\title{
Validation of the Spanish version of the physical activity questionnaire used in the Nurses' Health Study and the Health Professionals' Follow-up Study
}

\author{
Miguel Angel Martínez-González * , Constanza López-Fontana², José Javier Varo ${ }^{1}$, \\ Almudena Sánchez-Villegas ${ }^{1,3}$ and J Alfredo Martinez ${ }^{2}$ \\ ${ }^{1}$ Department of Epidemiology and Public Health, University of Navarra, Irunlarrea s/n, E-31080 Pamplona, Spain: \\ ${ }^{2}$ Department of Physiology and Nutrition, University of Navarra, Pamplona, Spain: ${ }^{3}$ Department of Clinical \\ Sciences, University of Las Palmas de Gran Canaria, Spain
}

Submitted 2 November 2004: Accepted 11 April 2005

\begin{abstract}
Objective: The objective of this analysis was to test the validity of the estimates of energy expenditure and sedentary lifestyle obtained through a self-administered questionnaire of physical activity for Spanish-speaking people adapted from US questionnaires (Nurses' Health Study and Health Professionals' Follow-up Study) using a triaxial accelerometer (RT3 Triaxial Research Tracker) as the reference.

Design and setting: Validation study, calculating the non-parametric correlation coefficients between the level of physical activity and sedentary lifestyle collected by the self-administered questionnaire and the triaxial accelerometer measurements. Percentage of misclassification and kappa coefficients were also calculated.

Subjects: The study population consisted of a sample of 40 obese women who were participants of the SUN (Seguimiento Universidad de Navarra) project (a prospective cohort study among Spanish university alumni). They were selected because of their peculiar metabolic characteristics, in the search for a sub-optimal scenario for validity. Results: Physical activity during leisure time (estimated as MET-h week ${ }^{-1}$ ) derived from the self-administered questionnaire moderately correlated with $\mathrm{kcalday}^{-1}$ assessed through the accelerometer (Spearman's $\rho=0.507,95 \%$ confidence interval (CI) $0.232,0.707)$. The Spearman correlation between the ratio of sedentary lifestyle to physical activity obtained through the questionnaire and the direct estimation (RT3) was -0.578 (95\% CI $-0.754,-0.325)$. The kappa index was $0.25(P=0.002)$ when assessing the cross-classification into quintiles and 0.41 for the dichotomous estimation of a sedentary lifestyle. Only $2.5 \%$ of participants were misclassified by the questionnaire more than two quintiles apart from the estimates of the RT3.

Conclusions: The moderate values obtained for correlation in a sub-optimal scenario for validity and the low percentage of extreme misclassification suggest the validity of the questionnaire to assess physical activity in Spanish-speaking women aged $20-50$ years.
\end{abstract}

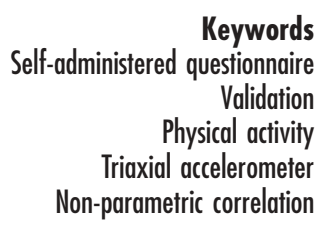

The influence of physical activity on several chronic diseases has been assessed in different epidemiological studies. Thus, physical activity has been associated with lower risk of coronary heart disease ${ }^{1}$, diabetes ${ }^{2}$, cancer $^{3,4}$ and other diseases and conditions such as the modification of glucose tolerance level, insulin sensitivity or lipid profile $^{5}$. An accurate measurement of physical activity in epidemiological research is crucial to determine its potential benefits on health.

Several different techniques have been used to assess physical activity, such as questionnaires, diaries, 7-day recall, movement sensors and doubly labelled water. Most of these methods calculate the energy expenditure associated with the activity. Questionnaires are the most widely used method to obtain information on physical activity, owing to their low cost, simplicity and briefness ${ }^{6}$.

The Nurses' Health Study and the Health Professionals' Follow-up Study used validated questionnaires to determine physical activity in large cohort studies based on North American populations ${ }^{7,8}$. However, no Spanish versions of these instruments have been validated so far.

The objective of the present analysis was to test the validity of the estimates of energy expenditure and sedentary lifestyle obtained through a self-administered physical activity questionnaire used in Spanish-speaking 
populations and previously adapted from US questionnaires $^{7,8}$.

\section{Subjects and methods}

The Spanish physical activity questionnaire used in this study has been adapted from those used in two large American cohorts, the Nurses' Health Study and the Health Professionals' Follow-up Study ${ }^{7,8}$. In those studies physical activity was determined through validated questionnaires mailed to participants.

The first part of the Spanish version of the questionnaire consisted of questions about participation in 17 different activities during leisure time (Fig. 1) and time spent on each (obtained through 10 categories ranging between 'never' and ' 11 or more hours per week') .
The second part of the questionnaire included questions about the number of hours spent in sedentary activities (watching television, sitting in front of a computer, driving, total time sitting, sleeping, sunbathing in summer, sunbathing in winter, going out with friends) and indicators of physical activity at work (standing, housework, work activities more intense than standing).

The number of months a year that every activity was performed was also asked, due to the seasonal practice of some activities (skiing, swimming, etc.). The options given were 'less than 3 months', ' 3 to 6 months' and 'more than 6 months'.

The number of METs (metabolic equivalents) corresponding to each activity were calculated using the Compendium of Physical Activities ${ }^{10}$ as the ratio of energy expended during a physical activity to the metabolic rate

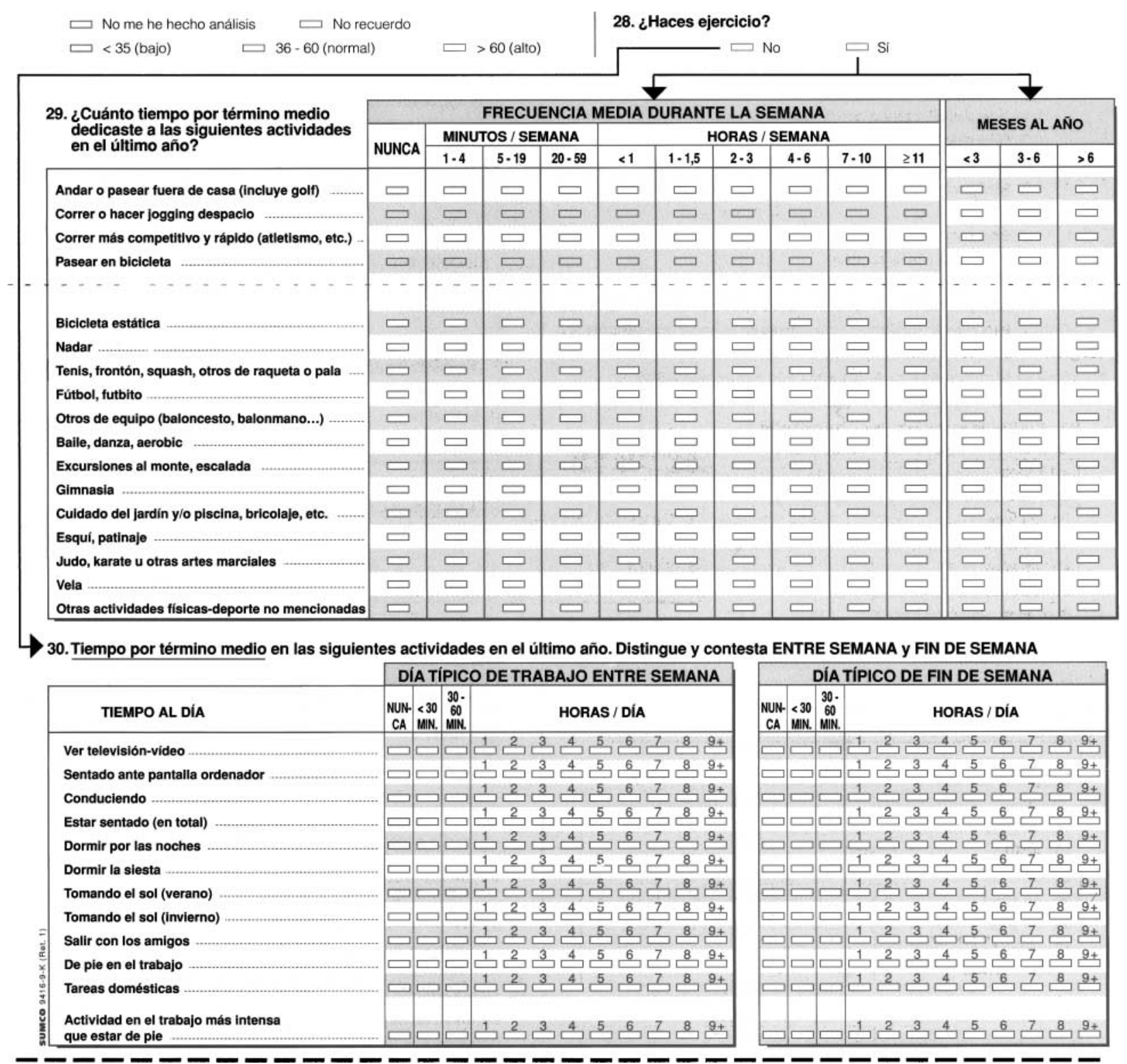

Fig. 1 Format and content of the self-administered questionnaire 
of sitting quietly. Afterwards, the number of METs in each activity was multiplied by the weekly participation in that activity $^{11,12}$. The estimated number of MET-h week ${ }^{-1}$ was weighted according to the number of months dedicated to each activity. Recreational physical activity was quantified by summing the MET-h week ${ }^{-1}$ dedicated to all activities performed during leisure time.

Total physical activity (MET-h week ${ }^{-1}$ ) was calculated by adding leisure-time physical activity to work physical activity.

A sedentary lifestyle index was calculated using the sedentary lifestyle indicators included in the questionnaire for a typical work day and for a typical weekend day. A sedentary lifestyle was defined based on the total number of hours spent sitting down per week ${ }^{12}$. The number of hours sitting down per week was estimated by multiplying by 5 the hours sitting down in a typical weekday and adding twice the average estimate for a typical weekend day. A relative index of sedentary lifestyle was calculated for each participant, assigning the $100 \%$ to the most sedentary participant and classifying the rest of the participants according to that value (sedentary lifestyle percentage). In the same way, the most active participant (MET-hweek $^{-1}$ ) was assigned $100 \%$ and the relative energy expenditure for the remaining participants was calculated as a proportion of this total (total activity percentage).

The ratio $\%$ sedentary lifestyle $\left(\mathrm{hweek}^{-1}\right) / \%$ total activity was also calculated and expressed as METh week $^{-1}$.

In the validation study of the Spanish physical activity questionnaire, a triaxial accelerometer was used as gold standard. The RT3 (Triaxial Research Tracker; Stayhealthy Inc., Monrovia, CA, USA) is a triaxial accelerometer consisting of three ceramic components orthogonally assembled. Once adjusted to the body, every time the subject moves, the RT3 objectively detects and measures the frequency and magnitude of accelerations and decelerations in $1 \mathrm{~min}$ intervals in three axis: forwards and backwards $(x)$, side to side $(y)$ and up and down $(z)$. The kinetic energy is converted into electrical energy transferred to the microprocessor, which translates it into vector magnitude (Vmag) and kilocalories. Using several equations, the vector values are converted into energy expenditure to calculate the physical activity energy expenditure $^{13}$.

Different studies have validated the results obtained with the triaxial accelerometer using the doubly labelled water method and with indirect calorimetry condition in humans under normal lifestyle ${ }^{14}$.

For the validation study, a sample of obese women with low educational level was selected, searching for a suboptimal scenario for validity given the fact that if validity was demonstrated in a sub-optimal scenario, it would be higher when applying the questionnaire to other populations with better basal conditions (higher between-subject variability and better understanding of the questionnaire). A sample of 40 obese women was selected (body mass index (BMI) $>29.5 \mathrm{~kg} \mathrm{~m}^{-2}$ ). Only $25 \%$ of them had attained college level of education, and $41 \%$ of them had achieved primary studies or less.

None of participants suffered from any known endocrine or metabolic disease, or were taking any drug known to affect the basal metabolic rate. All volunteers were selected according to the following inclusion criteria: being a woman, aged $20-50$ years, obese ${ }^{15}$ with BMI equal to or greater than $29.5 \mathrm{~kg} \mathrm{~m}^{-2}$, in premenopausal stage with regular menstruation and with stable weight ( $\pm 3 \mathrm{~kg}$ in the last 3 months).

Participants suffering from high blood pressure, diabetes mellitus, drug-treated hyperlipidaemia and/or thyroid disease, surgery-treated obesity, pregnant, with high intake of alcoholic drinks or drugs, or those who had participated in a clinical trial or nutritional intervention trial in the last 3 months were excluded from the study.

The participants were met to calibrate the RT3, put it on, and to explain to them how to use it. The accelerometer was put on the participant's waist and connected. From this moment the RT3 recorded all movements made until data were unloaded with the RT3 Assist Software, and the participant was unable to stop it. The device can store data for 2 weeks.

The participants wore the RT3 for 3 days in a typical week and 2 days at the weekend. They could take it off for sleeping at night and for hygiene.

All participants had previously completed the selfadministered physical activity questionnaire. The questionnaire was included in a group of questionnaires on diet, healthy habits and lifestyle. They were informed that the aim of the study was to evaluate with different methods their physical activity level and other aspects of diet and lifestyle, so they should keep their normal habits while wearing the device. However, they were not informed about the future correlation between their answers to the questionnaire and the data from the RT3.

Body composition was determined by anthropometric measures including weight, height, hip-to-waist ratio, body composition indices and bioimpedance measures. Mean (standard deviation, SD) BMI was 37.2 (6.1) $\mathrm{kg} \mathrm{m}^{-2}$.

\section{Statistical analysis}

Non-parametric Spearman correlation coefficients ( $\rho$ ) were calculated between energy expenditure estimation $\left(\mathrm{kcal}_{\mathrm{day}}{ }^{-1}\right.$ ) obtained with the reference device (triaxial accelerometer, RT3) and each the following variables from the questionnaire: total number of MET-h week ${ }^{-1}$, number of MET-h week ${ }^{-1}$ dedicated only to leisure-time physical activity, sedentary lifestyle index measured as total number of hours spent sitting down and also as the ratio \% sedentary lifestyle (h week $\left.{ }^{-1}\right) / \%$ total activity, as explained above.

Participants were categorised into quintiles of physical activity and sedentary lifestyle according to the 
measurements from the self-administered questionnaire and from the accelerometer (RT3). The percentage of misclassification due to the use of the questionnaire was also estimated. A participant was considered as misclassified if the difference in classification by questionnaire and by the gold-standard method was higher than two quintiles.

The agreement between the physical activity/sedentary lifestyle category obtained with the questionnaire and the energy expenditure obtained with the triaxial accelerometer was assessed by calculating the kappa coefficient for the cross-classification in quintiles according to both methods. In addition, medium- or high-intensity physical activity was considered if energy expenditure was in the two higher quintiles or the ratio \% sedentary lifestyle ( h week $^{-1}$ ) $\%$ total activity (MET-h week ${ }^{-1}$ ) obtained with the questionnaire was in the two lower quintiles. A woman was considered as sedentary if she was in the two lower quintiles of energy expenditure or in the two higher quintiles of the ratio $\%$ sedentary lifestyle $\left(\mathrm{h}_{\text {week }}{ }^{-1}\right) / \%$ total activity (MET-h week ${ }^{-1}$ ). Kappa indices were also estimated using these dichotomous classifications.

\section{Results}

The mean age of the volunteers was 34.3 (SD 7.1) years, with a maximum of 50 years and a minimum of 20 years. Table 1 shows the main characteristics associated with body composition of the participants.

Table 2 shows the estimations of physical activity obtained through the self-administered questionnaire and the triaxial accelerometer, and also the estimation of a sedentary lifestyle. The mean of MET-h week ${ }^{-1}$ during leisure time was 6.9 (SD 9.5), and 145.2 (SD 80.3) considering also work-time physical activity.

The physical activity energy expenditure measured with the accelerometer was 951.4 (SD 352.9) $\mathrm{kcal} \mathrm{day}^{-1}$. The mean sedentary lifestyle index was 61.9 (SD 31.4) $\mathrm{h}_{\text {week }}{ }^{-1}$.

Table 3 shows non-parametric Spearman correlation coefficients $(\rho)$ and their 95\% confidence intervals (CIs)

Table 1 Main characteristics associated with body composition (including anthropometric and bioimpedance measurements)

\begin{tabular}{lccc}
\hline Measurement & Mean & SD & Range \\
\hline Weight $(\mathrm{kg})$ & 94.7 & 15.9 & $69.1-137.4$ \\
Height $(\mathrm{m})$ & 1.60 & 0.006 & $1.48-1.79$ \\
Body mass index $\left(\mathrm{kg} \mathrm{m}^{-2}\right)$ & 37.15 & 6.08 & $29.83-56.46$ \\
Waist circumference $(\mathrm{cm})$ & 100.5 & 13.7 & $82.0-145.3$ \\
Hip circumference $(\mathrm{cm})$ & 121.7 & 13.7 & $102.7-159.9$ \\
Waist-to-hip ratio & 0.82 & 0.04 & $0.73-0.91$ \\
Triceps skinfold $(\mathrm{mm})$ & 36.6 & 3.6 & $28.2-43.0$ \\
Arm muscular perimeter $(\mathrm{cm})$ & 25.9 & 2.5 & $20.9-32.1$ \\
Arm muscular area $\left(\mathrm{cm}^{2}\right)$ & 53.7 & 10.7 & $34.7-82.2$ \\
Arm adipose area $\left(\mathrm{cm}^{2}\right)$ & 58.1 & 9.0 & $42.5-82.7$ \\
Adipose/muscular index & 1.10 & 0.17 & $0.73-1.71$ \\
\hline
\end{tabular}

SD - standard deviation. obtained between the possible combinations of physical activity and/or sedentary lifestyle obtained through the questionnaire and energy expenditure determined through the accelerometer (reference method).

We found a linear direct association between total MET$\mathrm{h}_{\text {week }}{ }^{-1}$ expended during leisure time obtained through the questionnaire and daily energy measured with the RT3 ( $\rho=0.507 ; 95 \%$ CI $0.232,0.707)$. When we analysed total physical activity, the Spearman coefficient was 0.451 (95\% CI 0.162, 0.669).

When we compared the sedentary lifestyle index with the energy expenditure measured with the RT3, the Spearman coefficient maintained the same value, although it showed an inverse association ( $\rho=-0.420$; $95 \%$ CI $-0.647,-0.125$ ). The coefficient for the association with the ratio \% sedentary lifestyle/\% total activity was $\rho=-0.578$ (95\% CI $-0.754,-0.325)$.

Table 4 shows the distribution of participants in each of the quintiles of the ratio \% sedentary lifestyle/\% total activity and in each of the quintiles of physical activity obtained through the accelerometer.

Forty per cent of the women were correctly classified by the physical activity questionnaire. Twenty five per cent of them were classified in the adjacent quintile compared with the objective measure of the RT3. Only $2.5 \%$ of participants were misclassified (i.e. classified two quintiles away from the measure obtained with the accelerometer).

The kappa coefficient for the concordance between the information obtained with the questionnaire and the reference method was $0.405(P=0.01)$ for the dichotomous estimation of a sedentary lifestyle (last two quintiles of the ratio \% sedentary lifestyle/\% total physical activity or first two quintiles of the reference energy expenditure). For the dichotomous estimation of moderate- and high-intensity physical activity (first two quintiles of the ratio $\%$ sedentary lifestyle/\% total physical activity or last two quintiles of the reference energy expenditure) the kappa coefficient was $0.286(P=0.069)$. Using the full cross-classification in quintiles (five categories for each measurement, Table 4), the kappa index was 0.249 $(P=0.002)$.

\section{Discussion}

This validation study in Spain of the self-administered questionnaires used in the widely known American cohorts (Nurses' Health Study, Health Professionals' Follow-up Study) offers some advantages. First, we have validated an instrument for its use in Spanish-speaking populations that have previous positive experience and have been widely used with success in physical activity epidemiology. Second, the reference method for the validation was a triaxial accelerometer, an objective method whose errors are not correlated with the errors of the questionnaire. Third, an acceptable correlation for leisure-time physical activity, total physical activity, for the sedentary lifestyle 
Table 2 Physical activity measures obtained with the self-administered questionnaire and the triaxial accelerometer

\begin{tabular}{|c|c|c|c|}
\hline Measurement & Mean & SD & Range \\
\hline Triaxial accelerometer $\left(\mathrm{kcal} \mathrm{day}^{-1}\right)$ & 951.4 & 352.9 & $337.5-1717.5$ \\
\hline Leisure-time physical activity (MET-h week ${ }^{-1}$ ), questionnaire & 6.9 & 9.5 & $0-5.0$ \\
\hline Total physical activity (MET-h week ${ }^{-1}$ ), questionnaire & 145.2 & 80.3 & $5.48-11.0$ \\
\hline Sedentary index $\left(\mathrm{h}^{2}\right.$ eek $\left.^{-1}\right)$, questionnaire & 61.9 & 31.4 & $11.5-28.5$ \\
\hline Ratio \% sedentary lifestyle/\% total activity, questionnaire & 10.5 & 13.0 & $0.0-3.8$ \\
\hline
\end{tabular}

SD - standard deviation; MET - metabolic equivalent.

index and for the ratio $\%$ sedentary lifestyle/\% total physical activity was found. Finally, this questionnaire can be used in large epidemiological studies consisting of consecutive measures since it can be answered quickly and requires little collaboration by the patient.

The correlations between the estimates obtained by the questionnaire and the objective measurement (triaxial accelerometer) for physical activity were similar to those found when validating food-frequency questionnaires ${ }^{16}$. To validate physical activity questionnaires in large American cohorts, daily records and 7-day recalls have been used instead of the triaxial accelerometer or other measurements that are highly independent of the ability of the patient to recall or to collaborate. The correlation coefficients between questionnaires and daily records for total moderate and vigorous physical activity were 0.62 in the Nurses' Health Study and 0.58 for vigorous activity and 0.28 for non-vigorous activity in the Health Professionals' Follow-Up Study ${ }^{17}$. Thus, the correlation coefficients found in our study are similar to the coefficients obtained in the validation studies conducted in the $\mathrm{USA}^{7,8}$.

The selection of a reference method in validation studies is crucial. The most accurate for determining physical activity energy expenditure is the doubly labelled water method, although its high cost and complexity make it unaffordable and infeasible for large studies.

It is essential that the errors of measure of the reference method are independent of the errors of the method in validation. The use of 7-day recalls and daily records has shown good results in several studies ${ }^{7,8,18}$, but they depend on the willingness to collaborate and/or memory of the participants, so they can show questionnaire-related measurement errors.

For all these reasons it is preferable to use a reference method that is as objective and independent as possible.
Some studies have shown the validity and reliability of data from movement sensors ${ }^{6}$, under both controlled conditions $^{19,20}$ and real conditions ${ }^{21}$. They have the advantage of being independent of memory and written records of the participants, and therefore their errors are quite independent of those of the questionnaire. However, so far, very few studies have validated physical activity frequency questionnaires by this method ${ }^{22-27}$.

In a previous validation study of a self-administered questionnaire to assess physical activity during a typical weekday, including sleeping, work and leisure time, no significant correlations were found between the number of METs and the activity measured with a triaxial accelerometer (Computer Science Application, CSA), either in men (Spearman's $\rho=0.05$ ) or women (Spearman's $\rho=0.31)^{22}$.

Nevertheless, Ainsworth et al $^{23}$ found a modest but significant $(P<0.001)$ correlation when comparing values from a uniaxial accelerometer (CSA) with estimations from a physical activity questionnaire for moderate-, high- or very high-intensity activities (Spearman's $\rho=0.30-0.33$ ).

The same results were found (around 0.30) in the validation study of the IPAQ (International Physical Activity Questionnaire) ${ }^{24}$. Validation and reliability of this questionnaire were performed through the collaboration of 14 participant centres from 12 different countries. Correlation coefficients were calculated for the practice of any physical activity and for a sedentary lifestyle, defined as the total number of hours spent sitting down. The objective measure in this case was a uniaxial accelerometer (CSA).

The correlations obtained in our study were higher than those mentioned above and also recently reported correlations in the USA, where a study used a uniaxial accelerometer as the reference method to validate physical

Table 3 Spearman correlation coefficients $(\rho)$ between measurements obtained with the questionnaire and the energy expenditure estimation (kcal day ${ }^{-1}$ ) from the reference method (triaxial accelerometer, RT3)

\begin{tabular}{lccr}
\hline & $\rho$ & $95 \% \mathrm{Cl}$ & $P$-value \\
\hline Recreational physical activity (MET-h week $^{-1}$ ) & +0.507 & $+0.232,+0.707$ & $<0.001$ \\
Total physical activity (MET-h week $^{-1}$ ) & +0.451 & $+0.162,+0.669$ & 0.003 \\
Sedentary index (h week $^{-1}$ ) & -0.420 & $-0.647,-0.125$ & 0.007 \\
Ratio \% sedentary lifestyle/\% total activity & -0.578 & $-0.754,-0.325$ & $<0.001$ \\
\hline
\end{tabular}

MET - metabolic equivalent. 
Table 4 Number of subjects classified in each quintile using the self-administered questionnaire and the reference method (triaxial accelerometer, RT3)

\begin{tabular}{lcccccr}
\hline $\begin{array}{l}\text { Physical } \\
\text { activity } \\
\text { quintile } \\
\begin{array}{l}\text { according } \\
\text { to the RT3 }\end{array}\end{array}$ & \multicolumn{5}{c}{$\begin{array}{l}\text { Quintile according to the questionnaire } \\
\text { (ratio \% sedentary lifestyle/\% total activity) }\end{array}$} \\
\cline { 2 - 7 } & Q5 & Q4 & Q3 & Q2 & Q1 & Total \\
\hline Q1 & 5 & 1 & 2 & & & 8 \\
Q2 & 1 & 2 & 1 & 2 & & 6 \\
Q3 & 1 & & 2 & 3 & 2 & 8 \\
Q4 & & 4 & 1 & 3 & 2 & 10 \\
Q5 & 7 & 1 & 2 & 1 & 4 & 8 \\
Total & 8 & 8 & 9 & 8 & 40 \\
\hline
\end{tabular}

Overall kappa index $=0.249(P=0.002)$.

activity daily records (coefficients between 0.15 and 0.24 for total physical activity $)^{25}$.

In a US study ${ }^{8}$ the correlation coefficients were lower for women with BMI $>25 \mathrm{~kg} \mathrm{~m}^{-2}$ (Spearman's $\rho=0.06-$ 0.08). In another sample of women ${ }^{25}$ with BMI $<25$ $\mathrm{kg} \mathrm{m}^{-2}$, correlation coefficients increased from $0.15-0.24$ to $0.23-0.28$. This is probably due to the fact that obese and overweight women are less likely to engage in vigorous physical activities, thus decreasing the betweensubject variability. If the between-subject variability decreases, the capability to detect correlation between methods also decreases. Consequently, our results must be interpreted with care as an underestimation of the true validity, taking into account that the selected sample represents a sub-optimal scenario to find high correlations.

On the other hand, several studies have suggested that triaxial accelerometers obtain better estimations than uniaxial accelerometers, since under normal conditions individuals make multidirectional movements ${ }^{14,20,28}$. This, together with the proper adaptation of the questionnaire to the Spanish population, is one reason that could explain our good results.

Although the RT3 has been used to validate a telephone version of a 7-day recall ${ }^{29}$, to our knowledge this is the first study validating a self-administered questionnaire by using a triaxial accelerometer. The validation of the 7-day recall found higher correlations for physically intense or very intense activities. The correlation coefficients between the 7-day recall and the estimations of the triaxial accelerometer were 0.41 for total activity, 0.33 for moderate activity, 0.43 for intense activity and 0.74 for very intense activity ${ }^{29}$.

The low level of physical activity during leisure time observed in the women of the present validation sample is somewhat surprising. Their occupations were sedentary. In addition, the level of leisure-time physical activity in Spanish women is low relatively compared with other European countries as shown by comparative studies ${ }^{11}$. Among women with higher physical activity during leisure time, we would very likely have found better measure- ment and classification by the questionnaire because of increased between-subject variability. Therefore, obese sedentary women represent a sub-optimal scenario for validity, because of the lower between-subject variability that is usually found among them. Twenty-five per cent of the participants in our sample had attained college level of education and it is likely that validity may be higher among adults with a higher educational level. However, this questionnaire is currently used in the SUN cohort, where all participants have a very high level of education ${ }^{9,30,31}$.

Another possible limitation of our study is that the sample was limited to adult women aged $20-50$ years, and we acknowledge that there is no Spanish validation for men, children or women over 50 years old. This fact limits the generalisability of our findings to other demographic groups.

Further limitations can derive from different ways of using the device. The accelerometer was placed on the waist, and this could represent a potential problem for activities requiring mainly waist movement. Some authors criticise the use of accelerometers to estimate energy expenditure, arguing that accelerometers underestimate energy expenditure from low-intensity activities or from activities related with static exercise (weightlifting, exercise bike, water activities or movements of the upper limbs) $)^{20,32-34}$. Leenders et al. ${ }^{32}$ suggested that the mean energy expenditure from physical activity measured with triaxial (RT3) or uniaxial devices was 35-59\% lower than the estimation of the doubly labelled water method. However, the same authors consider them suitable for physical activity patterns that cannot be measured with doubly labelled water.

Although participants were instructed to wear the accelerometer for the whole day (except for sleeping and hygiene), we cannot exclude failure to comply with the instructions, which would lead to an underestimation of physical activity. Nevertheless, mistakes correlated with questionnaires of frequency and length of activity are less frequent among objective measures of physical activity obtained with a triaxial accelerometer, thus representing an ideal reference method against which to compare the questionnaire ${ }^{35,36}$.

A recent review has analysed the validity and reliability of values obtained through seven different auto-referred physical activity measurements. Reliability coefficients ranged between 0.34 and 0.89 , and validity coefficients were 0.14 to $0.53^{37}$. Therefore our estimations are acceptable (kappa index $=0.405$ and Spearman's $\rho=-0.578$ for the ratio $\%$ sedentary lifestyle $/ \%$ total physical activity).

In conclusion, the results of this analysis demonstrate the validity of this self-administered questionnaire about physical activity in Spanish-speaking women aged 20-50 years. The estimates reported here support the use of this questionnaire in epidemiological studies that include Spanish-speaking subjects. 


\section{Acknowledgement}

This work was supported by a grant from the Instituto de Salud Carlos III (Thematic Network about Diet and Cardiovascular Disease, grant G03/140 and FIS Project, grant PI040233), by the Health Department of the Government of Navarra and by the Special Research Line on Nutrition, Obesity and Health of the University of Navarra.

\section{References}

1 Tanasescu M, Leitzmann MF, Rimm EB, Willett WC, Stampfer MJ, Hu FB. Exercise type and intensity in relation to coronary heart disease in men. Journal of the American Medical Association 2002; 288: 1994-2000.

2 Kriska AM, Saremi A, Hanson RL, Bennett PH, Kobes S, Williams DE, et al. Physical activity, obesity, and the incidence of type 2 diabetes in a high-risk population. American Journal of Epidemiology 2003; 158: 669-75.

3 McTiernan A, Kooperberg C, White E, Wilcox S, Coates R, Adams-Campbell LL, et al.; Women's Health Initiative Cohort Study. Recreational physical activity and the risk of breast cancer in postmenopausal women: the Women's Health Initiative Cohort Study. Journal of the American Medical Association 2003; 290: 1331-6.

4 Mao Y, Pan S, Wen SW, Johnson KC; Canadian Cancer Registries Epidemiology Research Group. Physical activity and the risk of lung cancer in Canada. American Journal of Epidemiology 2003; 158: 564-75.

5 Varo Cenarruzabeitia JJ, Martínez Hernández JA, MartínezGonzález MA. Beneficios de la actividad física y riesgos del sedentarismo. Medicina Clinica 2003; 121: 665-72.

6 Delgado Rodríguez M, Martínez-González MA, Aguinaga Ontoso I. Actividad física y salud. In: Gálvez Vargas R, Sierra López A, Saénz González MC, et al., eds. Piédrola Gil. Medicina Preventiva y Salud Pública. 10th ed. Barcelona: Masson, 2000; 935-44.

7 Chasan-Taber S, Rimm EB, Stampfer MJ, Spiegelman D, Colditz GA, Giovannucci E, et al. Reproducibility and validity of a self-administered physical activity questionnaire for male health professionals. Epidemiology 1996; 7: $81-6$.

8 Wolf AM, Hunter DJ, Colditz GA, Manson JE, Stampfer MJ, Corsano KA, et al. Reproducibility and validity of a selfadministered physical activity questionnaire. International Journal of Epidemiology 1994; 23: 991-9.

9 Sánchez-Villegas A, Martínez-González MA, Toledo E, de Irala-Estevez J, Martinez JA for the SUN Project. Influencia del sedentarismo y el hábito de comer entre horas sobre la ganancia de peso. Medicina Clinica 2002; 119: 46-52.

10 Ainsworth BE, Haskell WL, Whitt MC, Irwin ML, Swartz AM, Strath SJ, et al. Compendium of physical activities: an update of activity codes and MET intensities. Medicine and Science in Sports and Exercise 2000; 32(Suppl. 9): S498-S504.

11 Martínez-González MA, Varo JJ, Santos JL, De Irala-Estévez J, Gibney M, Kearney J, et al. Prevalence of physical activity during leisure time in the European Union. Medicine and Science in Sports and Exercise 2001; 33: 1142-6.

12 Martínez-Gonzalez MA, Martínez JA, Hu FB, Gibney MJ, Kearney J. Physical inactivity, sedentary lifestyle and obesity in the European Union. International Journal of Obesity and Related Metabolic Disorders 1999; 23: 1-10.

13 Tudor-Locke CE, Myers AM. Challenges and opportunities for measuring physical activity in sedentary adults. Sports Medicine 2001; 3: 91-100.
14 Westerterp KR. Physical activity assessment with accelerometers. International Journal of Obesity and Related Metabolic Disorders 1999; 23: S45-9.

15 World Health Organization (WHO). Obesity: Preventing and Managing the Global Epidemic. WHO Consultation on Obesity. Technical Report Series No. 894. Geneva: WHO, 1998.

16 Martin-Moreno JM, Boyle P, Gorgojo L, Maisonneuve P, Fernandez-Rodriguez JC, Salvini S, et al. Development and validation of a food frequency questionnaire in Spain. International Journal of Epidemiology 1993; 22: 512-9.

17 Rosner B, Wolf AM, Willett WC. Correcting interclass correlation coefficients for measurement error in unbalanced data sets [abstract]. American Journal of Epidemiology 1993; 138: 597

18 Sallis JF, Haskell WL, Wood PD, Fortmann SP, Rogers T, Blair $\mathrm{SN}$, et al. Physical activity assessment methodology in the Five-City Project. American Journal of Epidemiology 1985; 121: $91-106$.

19 Welk GJ, Almeida J, Morss G. Laboratory calibration and validation of the Biotriner and Actitrac activity monitors. Medicine and Science in Sports and Exercise 2003; 35 1057-64.

20 Bouten CV, Westerterp KR, Verduin M, Janssen JD. Assessment of energy expenditure for physical activity using a triaxial accelerometer. Medicine and Science in Sports and Exercise 1994; 26: 1516-23.

21 Levine JA, Baukol PA, Westerterp KR. Validation of the Tracmor triaxial accelerometer system for walking. Medicine and Science in Sports and Exercise 2001; 33: 1593-7.

22 Aadahl M, Jorgensen T. Validation of a new self-report instrument for measuring physical activity. Medicine and Science in Sports and Exercise 2003; 35: 1196-202.

23 Ainsworth BE, Bassett DR, Strath SJ, Swartz AM, O'Brien WL, Thompson RW, et al. Comparison of three methods for measuring the time spent in physical activity. Medicine and Science in Sports and Exercise 2000; 32: S457-64.

24 Craig CL, Marshall AL, Sjostrom M, Bauman AE, Booth ML, Ainsworth BE, et al. International physical activity questionnaire: 12-country reliability and validity. Medicine and Science in Sports and Exercise 2003; 35: 1381-95.

25 Schmidt MD, Freedson PS, Chasan-Taber L. Estimating physical activity using the CSA accelerometer and a physical activity log. Medicine and Science in Sports and Exercise 2003; 35: 1605-11.

26 Miller DJ, Freedson PS, Kline GM. Comparison of activity levels using the Caltrac accelerometer and five questionnaires. Medicine and Science in Sports and Exercise 1994; 26: 376-82.

27 Pereira MA, FitzerGerald SJ, Gregg EW, Joswiak ML, Ryan WJ, Suminski RR, et al. A collection of physical activity questionnaires for health-related research. Medicine and Science in Sports and Exercise 1997; 29: S1-205.

28 Fogelholm M, Hiilloskorpi H, Laukkanen R, Oja P, Van Marken Lichtenbelt W, Westerterp K. Assessment of energy expenditure in overweight women. Medicine and Science in Sports and Exercise 1998; 30: 1191-7.

29 Hayden-Wade HA, Coleman KJ, Sallis JF, Armstrong C. Validation of the telephone and in-person interview versions of the 7-day PAR. Medicine and Science in Sports and Exercise 2003; 35: 801-9.

30 Martinez-Gonzalez MA, Sanchez-Villegas A, De IJ, Marti A, Martinez JA. Mediterranean diet and stroke: objectives and design of the SUN project. Seguimiento Universidad de Navarra. Nutritional Neuroscience 2002; 5: 65-73.

31 Alonso A, de la Fuente C, Martin-Arnau AM, de Irala J, Martinez JA, Martinez-Gonzalez MA. Fruit and vegetable consumption is inversely associated with blood pressure in a Mediterranean population with a high vegetable-fat intake: the Seguimiento Universidad de Navarra (SUN) Study. British Journal of Nutrition 2004; 92: 311-9. 
32 Leenders NY, Sherman WM, Nagaraja HN, Kien CL. Evaluation of methods to assess physical activity in freeliving conditions. Medicine and Science in Sports and Exercise 2001; 33: 1233-40.

33 Chen KY, Sun M. Improving energy expenditure estimation by using a triaxial accelerometer. Journal of Applied Physiology 1997; 83: 2112-22.

34 Hendelman D, Miller K, Baggett C, Debold E, Freedson P. Validity of accelerometry for the assessment of moderate intensity physical activity in the field. Medicine and Science in Sports and Exercise 2000; 32: S442-9.
35 Willett WC. Nutritional Epidemiology, 2nd ed. New York: Oxford University Press, 1998; 110.

36 Jakicic JM, Polley BA, Wing RR. Accuracy of self-reported exercise and the relationship with weight loss in overweight women. Medicine and Science in Sports and Exercise 1998; 30: 634-8.

37 Sallis JF, Saelens BE. Assessment of physical activity by self-report: status, limitations, and future directions. Research Quarterly for Exercise and Sport 2000; 71: $\mathrm{S} 1-14$. 